\title{
Florística e estrutura do componente arbustivo-arbóreo de um remanescente de cerrado sensu stricto, Gurupi, Tocantins
}

\section{Floristic and structure of the shrub-arboreal component of a remnant of cerrado sensustricto, Gurupi, Tocantins, Brazil}

\author{
Bruno Aurélio Campos Aguiar ${ }^{*}$, Marília Oliveira Camargo ${ }^{2}$, Rômullo Quirino de Souza Ferreira ${ }^{3}$, Paulo Ricardo Teixeira ${ }^{4}$, \\ Rubens Ribeiro da Silva ${ }^{5}$ Priscila Bezerra de Souza
}

Resumo: Objetivou-se caracterizar a florística e a fitossociologia de um fragmento de cerrado sensu stricto, localizado em Gurupi, Tocantins. O estudo foi realizado na Reserva legal da fazenda experimental da Universidade Federal do Tocantins, campus de Gurupi, Tocantins. Foram implantadas cinco parcelas de 20x50m, totalizando $5000 \mathrm{~m}^{2}$ e amostrados todos os indivíduos com circunferência maior ou igual a $10 \mathrm{~cm}$, ao nível do solo (CAS). Foram registrados 906 indivíduos arbustivoarbóreo pertencentes a 42 famílias, 102 espécies e 78 gêneros. As famílias que se destacarem em numero de indivíduos, frequência, dominância foram Myrtacea, Vochystaceae, Burseraceae e Fabaceae, entre os indivíduos os principais foram Myrcia fallax (Rich) DC, Protium heptaphyllum (Aubl) Marchand e Qualea multiflora Mart. O índice de diversidade de Shannon $\left(\mathrm{H}^{\prime}\right)$ foi de 3,70 e equabilidade $\mathrm{J}^{\prime} 0,80$, sendo considerados padrões para a região do cerrado.

Palavras-chave: Levantamento florístico; Diversidade; Riqueza.

Abstract: The objective was to characterize a floristic and phytosociology of a fragment of cerrado sensu stricto, located in Gurupi, Tocantins. The study was carried out in the legal Reserve of the experimental farm of the Federal University of Tocantins, Campus of Gurupi, Tocantins, Brazil. Five porcelains of 20x50m were implanted, totaling 5000sqm and all compostations with a circumference greater than or equal to $10 \mathrm{~cm}$, at $0 \mathrm{~cm}$ at the soil level (CAS) were implanted. Foramaler 906 aphid shrub-arboreal belonging to 42 families, 102 species and 78 genera. The families that stood out in number of individuals, frequency, dominance were Myrtacea, Vochystaceae, Burseraceae and Fabaceae, among the indications of the main beings Myrcia fallax (Rich) DC, Protium heptaphyllum (Aubl) Marchand and Qualea multiflora Mart. The diversity index of Shannon $\left(\mathrm{H}^{\prime}\right)$ was 3.70 and equability $\mathrm{J}^{\prime} 0,80$, being considered for the cerrado region.

Key words: Floristic survey; Diversity; Wealth.

\footnotetext{
*Autor para correspondência

Recebido para publicação em 22/09/2017; aprovado em 22/12/2017

${ }^{1}$ Eng. Florestal, Mestrando do programa Ciências Florestais e Ambientais, Universidade Federal do Tocantins, Gurupi, aguiar.florestal@ gmail.com.

${ }^{2}$ Eng. Florestal, Mestre em Ciências Florestais e Ambientai, Universidade Federal do Tocantins, Gurupi, lilikamargo@ hotmail.com

${ }^{3}$ Eng. Florestal, Mestre em Ciências Florestais e Ambientais, Universidade Federal do Tocantins, Gurupi, romullo_florest@mail.uft.edu.br

${ }^{4}$ Eng. Florestal, Mestre em Ciências Florestais e Ambientais, Universidade Federal do Tocantins, Gurupi, paulo.engflorestal@ hotmail.com

${ }^{5}$ Eng. Agrônomo, Dr. Professor Adjunto do Curso de Agronomia e do Programa de Pós Graduação Ciências Florestais e Ambientais, Universidade Federal do Tocantins, Gurupi, rrs2002@uft.edu.br

${ }^{6}$ Bióloga, Dr. Professora Adjunto do Curso de Engenharia Florestal e do Programa de Pós Graduação Ciências Florestais e Ambientais, Universidade Federal do Tocantins, Gurupi, priscilauft@uft.edu.br
} 


\section{INTRODUÇÃO}

Dentre os biomas presente no Brasil, o Cerrado é o segundo maior bioma perdendo apenas para a Amazônia, ocupando cerca de $22 \%$ do território (BRASIL, 2017). Este bioma ocupa a área central do Brasil e engloba vários Estados, entre eles Goiás, Distrito Federal e parte dos Estados de Minas Gerais, Rondônia, Mato Grosso, Mato Grosso do Sul, Bahia, São Paulo Tocantins, Maranhão, Piauí e Pará (SANO et al., 2008).

O cerrado é composto por uma vegetação desde savanas, campos abertos e florestas, mudanças de fisionomias são oriundas principalmente devido à disponibilidade de água e outras características referente ao solo (SILVA et al.,2015).

A flora do bioma Cerrado é composta basicamente pelas mesmas famílias e gêneros que ocorrem na Mata Atlântica e na Amazônia, mas as espécies são distintas e com distribuição heterogênea dentro do bioma, além das proporções diferentes dos gêneros e espécies (RATTER et al., 2003; BRIDGEWATER et al., 2004). Adicione-se a isso, conforme Forzza et al. (2010), que $29,7 \%$ das plantas com sementes endêmicas do Brasil, ou 4.151 espécies, pertencem ao bioma Cerrado.

O bioma Cerrado já pode ser considerado um ambiente tropical que detêm elevada parcela de conhecimento sobre sua vegetação e chama atenção aos possíveis processos responsáveis pelos padrões ecológicos até agora identificados. Entretanto, para desenvolver essa compreensão acerca desses padrões (por exemplo, a elevada $\beta$-diversidade), é necessário dar continuidade a coleta de dados florísticos, assim como direcionar esforços para estudos de cunho fitogeográfico (DURIGAN et al., 2003; BRIDGEWATTER et al., 2004; FELFILI et al.,2004; NETTESHEIM et al., 2010; SOUZA FERREIRA et al., 2015).

O Estado do Tocantins possui $27.620 \mathrm{~km}^{2}$ de extensão territorial, tendo $91 \%$ composto pelo Bioma Cerrado (MACHADO et al., 2004). Diante do contexto apresenta ainda $72 \%$ da vegetação remanescente do bioma Cerrado, configurando-se entre os Estados com maiores índices desse tipo de cobertura (BRASIL, 2015)

Mesmo apresentando índices elevados desse tipo de vegetação o numero de estudos voltados para se conhecer a florística e fitossociologia da flora no Estado do Tocantins ainda é rara (BONFIM et al., 2016). Diante do contexto objetivou-se caracterizar a florística e a fitossociologia de um fragmento de cerrado sensu stricto, Gurupi-TO.

\section{MATERIAL E MÉTODOS}

A área de estudo é um fragmento do Cerrado, fisionomia cerrado sensu stricto, pertencendo a Fazenda Experimental da Universidade Federal do Tocantins, campus de Gurupi - TO, sob as coordenadas UTM $11^{\circ} 46^{\prime} 25^{\prime}$ ' S e $49^{\circ} 02^{\prime} 54^{\prime \prime}$ W. O clima da região é caracterizado como tropical úmido com estação chuvosa no verão e seca no inverno. A temperatura média ao longo do ano varia entre $22^{\circ} \mathrm{C}$ e $28^{\circ} \mathrm{C}$, já a precipitação varia entre $1.500 \mathrm{~mm}$ a $1.600 \mathrm{~mm}$ (SEPLAN, 2012). A altitude registrada nas áreas avaliadas está situada entre as cotas de 250 a 300 metros e o solo é classificado como Plintossolo (EMBRAPA, 2013).

A vegetação na área estudada foi avaliada entre os meses de setembro de 2012 a de agosto de 2013, utilizando-se o método de parcelas (MUELLER-DOMBOIS;
ELLENBERG, 1974). Foram instaladas sistematicamente cinco parcelas de $20 \times 50 \mathrm{~m}$, sendo distanciadas $20 \mathrm{~m}$ entre si, perfazendo um total de $5000 \mathrm{~m}^{2}$, ou seja, 0,5 ha de área amostral.

Nas parcelas foram amostrados todos os indivíduos arbustivo-arbóreo com circunferência ao nível do solo $\geq 10 \mathrm{~cm}$ (CAS).

A identificação taxonômica das espécies amostradas na área de estudo foi realizada através de comparações com o material do Herbário da UFT, campus de Porto Nacional, literatura especializada Almeida et al. (1998); Lorenzi (2002); Silva Junior (2009); Silva Junior (2012) e consultas a especialistas, quando necessário. Os nomes das espécies, seus respectivos autores e sinonímias foram confirmados e atualizados pelo site da Lista de Espécies da Flora do Brasil (FLORA DO BRASIL, 2015). O sistema de classificação adotado foi o "Angiosperm Phylogeny Group" (APG, 2009).

A partir do uso do programa Fitopac versão 2.1.2 foram obtidos os parâmetros fitossociológicos densidade relativa (DR), densidade absoluta (DA), dominância absoluta (DoA), dominância relativa (DoR), frequência absoluta (FA), frequência relativa (FR), índice de valor de importância (IVI), índice de diversidade de Shannon (H'), e Pielou (SHEPPERD, 2010).

\section{RESULTADOS E DISCUSSÃO}

Foram amostrados 906 indivíduos, dos quais 868 vivos e 38 mortos em pé, distribuídos em 102 espécies, 78 gêneros pertencentes a 42 famílias.

As árvores mortas em pé, totalizaram 4,19\% dos indivíduos amostrados, valor esse considerado normal em formações vegetais brasileiras. Como apresentou elevada frequência, ocorrendo em $100 \%$ das parcelas, indica que não está havendo uma perturbação localizada, pois a morte das árvores pode estar relacionada com acidentes como: ventos, tempestades, queda de grandes ramos, doenças, perturbações antrópicas e até mesmo ocorrer naturalmente por estarem velhas. Em relação ao total de árvores amostradas, outros autores encontraram valores parecidos, em áreas de Cerrado no Estado de São Paulo, Cavassan (1982) encontrou 5,8\% de árvores mortas, Struffaldi-De-Vuono (1985) 11,5\%, Martins (1991) 7,4\%, Tabanez et al. (1997) $11,3 \%$ e Silva e Soares (2002) $7,74 \%$. As árvores mortas, ainda em pé, têm valor ecológico para a fauna silvestre, fornecendo abrigo, local de nidificação e fonte indireta de alimento (LOPES, 2002).

As famílias com os maiores números de espécies foram Fabaceae (14), Myrtaceae (7), Annonaceae (6), Rubiaceae (6), Apocynaceae e Chrysobalanaceae com cinco espécies cada (Tabela 1). Estas famílias contribuíram com $42,15 \%$ do total das espécies amostradas, em se tratando da flora lenhosa de cerrado sensu stricto, as famílias como Fabaceae, Vochysiaceae, Myrtaceae, Malpighiaceae e Rubiaceae comumente apresentam altos valores de riqueza florística (FINA; MONTEIRO, 2013; NETTESHEIM et al., 2010; SILVA NETO et al., 2016; ROCHA et al., 2014)

A posição de destaque da Fabaceae entre as famílias com maior riqueza de espécies podem estar relacionadas com a capacidade de fixação de nitrogênio de algumas de suas espécies, o que pode conferir à referida família vantagem competitiva nos solos predominantemente distróficos do Cerrado (GOODLAND, 1979). Além disso, a grande representatividade da família Fabaceae pode ser atribuída ao 
fato da região central do Brasil ser considerada o principal centro de diversificação desta família (POLHILL et al.,1981).

As famílias Myrtaceae, Vochysiaceae, Burseraceae, Fabaceae, Anacardiaceae, Sapindaceae, Malpighiaceae, Tiliaceae, Polygonaceae, Annonaceae e Proteaceae obtiveram $100 \%$ de frequência absoluta, indicando uma ampla distribuição dessas famílias na área. Dados estes que corroboram com Neri et al. (2007) onde encontraram as famílias Vochysiaceae, Fabaceae e Malpighiaceae com 100\% de frequência em um levantamento realizado no cerrado do norte de Minas Gerais. Já as famílias Sapotaceae, Styracaceae, Melastomaceae, Boraginaceae, Opiliaceae, Celastraceae, Rutaceae, Lythraceae, Cochlospermaceae e Euphorbiaceae tiveram presentes em apenas uma das parcelas com frequência absoluta de $20 \%$.

As quatro famílias que apresentaram maiores índice de valor de importância foram Myrtaceae (9,84\%); Vochysiaceae (8,62\%); Burseraceae $(7,77 \%)$ e Fabaceae $(6,90 \%)$. Tal característica implica na dominância no nível de família, fato comum em regiões tropicais, como no cerrado sensu stricto.
Em relação ao número de indivíduos as famílias que obtiveram maior representação foram Myrtaceae (165), Vochysiaceae (131), Burseraceae (63), Fabaceae (58), Malpighiaceae (54) e Anacardiaceae (49), totalizando 57,39 $\%$ dos indivíduos amostrados.

O sucesso da família Vochysiaceae pode estar fortemente associado à capacidade de acumulação de alumínio por parte de algumas das suas espécies (HARIDASAN; ARAÚJO, 1987; HARIDASAN, 2000), o que também configura uma vantagem competitiva em solos distróficos com baixa saturação de bases trocáveis, baixa capacidade de troca catiônica, alta acidez e altas concentrações de $\mathrm{Al}$ no perfil, como é o caso da maioria dos solos no bioma Cerrado. Além disso, muitas espécies das referidas famílias têm estratégias reprodutivas bem adaptadas às condições climatológicas do Cerrado, apresentando dispersão das sementes no início da estação chuvosa, boa germinação e ausência de dormência nas sementes (OLIVEIRA, 2008).

Tabela 1. Estimativa dos parâmetros fitossociológicos das famílias e do grupo das árvores mortas, na área de cerrado sensu stricto. Ao nível de amostragem a $0 \mathrm{~cm}$ do solo (CAS), ordenadas em ordem decrescente de valor de importância, em que NI= número de indivíduos; $\mathrm{NSp}=$ número de espécies; $\mathrm{DA}=$ densidade absoluta (número de indivíduos/ha); DR = densidade relativa $(\%)$; FrA = frequência absoluta (unidade de amostra/total de amostra); FrR = frequência relativa $(\%)$; DoA = dominância absoluta ( $\left.\mathrm{m}^{2} / \mathrm{ha}\right)$; DoR = dominância relativa(\%) e IVI = índice de valor de importância $(\%)$.

\begin{tabular}{lccccccccc}
\hline \multicolumn{1}{c}{ Famílias } & NI & NSp & DA & DR & FrA & FrR & DoA & DoR & IVI\% \\
\hline Myrtaceae & 165 & 7 & 330 & 18,21 & 100 & 3,65 & 2,84 & 7,64 & 9,84 \\
Vochysiaceae & 131 & 4 & 262 & 14,46 & 100 & 3,65 & 2,88 & 7,76 & 8,62 \\
Burseraceae & 63 & 1 & 126 & 6,95 & 100 & 3,65 & 4,72 & 12,71 & 7,77 \\
Fabaceae & 58 & 14 & 116 & 6,40 & 100 & 3,65 & 3,95 & 10,65 & 6,90 \\
Anacardiaceae & 49 & 4 & 98 & 5,41 & 100 & 3,65 & 3,56 & 9,59 & 6,22 \\
Morta & 38 & 1 & 76 & 4,19 & 100 & 3,65 & 2,65 & 7,15 & 5,00 \\
Sapindaceae & 48 & 2 & 96 & 5,30 & 100 & 3,65 & 2,21 & 5,96 & 4,97 \\
Malpighiaceae & 54 & 4 & 108 & 5,96 & 100 & 3,65 & 0,84 & 2,27 & 3,96 \\
Tiliaceae & 18 & 1 & 36 & 1,99 & 100 & 3,65 & 1,18 & 3,19 & 2,94 \\
Rubiaceae & 29 & 6 & 58 & 3,20 & 80 & 2,92 & 0,95 & 2,57 & 2,90 \\
Combretaceae & 15 & 1 & 30 & 1,66 & 80 & 2,92 & 1,46 & 3,94 & 2,84 \\
Bombacaceae & 10 & 2 & 20 & 1,10 & 80 & 2,92 & 1,60 & 4,32 & 2,78 \\
Chrysobalanaceae & 22 & 5 & 44 & 2,43 & 80 & 2,92 & 1,11 & 2,98 & 2,78 \\
Polygonaceae & 25 & 1 & 50 & 2,76 & 100 & 3,65 & 0,69 & 1,86 & 2,75 \\
Annonaceae & 23 & 6 & 46 & 2,54 & 100 & 3,65 & 0,68 & 1,83 & 2,67 \\
Loganiaceae & 19 & 1 & 38 & 2,10 & 80 & 2,92 & 1,05 & 2,83 & 2,61 \\
Apocynaceae & 19 & 5 & 38 & 2,10 & 80 & 2,92 & 0,61 & 1,64 & 2,22 \\
Proteaceae & 14 & 1 & 28 & 1,55 & 100 & 3,65 & 0,42 & 1,14 & 2,11 \\
Icacinaceae & 18 & 1 & 36 & 1,99 & 80 & 2,92 & 0,53 & 1,42 & 2,11 \\
Outras espécies & 88 & 35 & 176 & 9,68 & 980 & 35,77 & 3,16 & 8,56 & 18,01 \\
\hline Total & 906 & 102 & 1812 & 100 & 2740 & 100 & 37,09 & 100 & 100 \\
\hline
\end{tabular}

As espécies com maior número de indivíduos foram Myrcia fallax (137), Qualea multiflora (68) Protium heptaphyllum (63), Magonia pubescens (42), Qualea grandiflora (31),Byrsonima pachyphylla (28), Tapirira guianensis (28) e Qualea parviflora (28), totalizando 46,90\% dos indivíduos amostrados (Tabela 2).

Excluindo as mortas treze espécies de diferentes famílias apresentaram $100 \%$ de frequência absoluta, sendo comuns em todas as cinco parcelas entre elas estão Myrcia fallax, Protium heptaphyllum, Qualea multiflora, Tapirira guianensis, Magonia, pubescens, Qualea parviflora, Luehea grandiflora, Copaifera langsdorffii, Coccoloba mollis, Vatairea macrocarpa, Xylopia aromatica, Astronium fraxinifolium e
Roupala montana no entanto as quatro últimas espécies apresentaram poucos indivíduos, apesar da alta frequência (Tabela 2).

As espécies que apareceram em apenas uma das parcelas foram Platypodium elegans, Hirtella martiana, Hirtella gracilipes, Gomidesia lindeniana, Sclerolobium aureum, Brosimum gaudichaudii, Coussarea hydrangeaefolia, Psychotria carthagenensis, Byrsonima laxiflora, Davilla elliptica, Aspidosperma macrocarpo, Pouteria ramiflora, Psidium myrsines, Agonandra brasiliensis, Styrax ferrugineus, Rapanea gardneriana, Anona crasiflora, Anacardium occidentale, Mouriripusa, Psidium guineense, Guatteria nigrescens, Alibertia edulis, Cordia glabrata, 
Guatteria vilosíssima, Ficus insipida, Hymenaea courbaril, Ascomium dasycarpum, Guarea macrophylla, Tachigali aurea, Machaerium opacum, Porcelia macrocarpa, Maytenus robusta, Tabebuia ocrhacea, Diospyros burchelli, Zanthoxylum rhoifolium, Licania humilis, Physocalym mascaberrimum, Cybianthus detergens, Harcornias peciosa, Brosimum guianense, Aspidosperma ramiflora, Connarus suberosus, Guarea guidonia, Rourea induta, Aspidosperma parvifolium, Cochlospermum regium, Hyeronima alchorneoides, Ouratea hexasperma e Bauhinia rufa, totalizando 48,03\% das espécies amostradas.

Dessas espécies citadas acima 37 apareceram com apenas um individuo, totalizando $36,27 \%$ das espécies encontradas no fragmento estudado. Cabe ressaltar que é um número muito elevado de espécies que ocorrem com apenas um indivíduo, indicando uma alta susceptibilidade à extinção local dessas espécies, caso ocorra morte ou corte desses indivíduos. Isso pode ser devido à grande diversidade da flora, ao padrão de distribuição da espécie e a baixa densidade das populações (SILVA e SOARES, 2002).

As espécies que mostraram maior dominância relativa foram Protium heptaphyllum (12,71\%), Tapirira guianensi $s(7,93 \%)$, Myrcia fallax (5,69\%) e Magonia pubescens $(5,09 \%)$.

Com relação ao índice de valor de importância destacaram-se as espécies Myrcia fallax (7,67\%), Protium heptaphyllum (7,29\%), Qualea multiflora (4,54\%), Tapirira guianensis (4,41\%) e Magonia pubescens (3,97\%). Um grande número de espécies $(68,62 \%)$ apresentaram IVI menor que 1\% (Tabela 2). Segundo Martins (1979), a presença de um grande número de espécies com baixo IVI é uma característica das florestas tropicais. Poucas espécies detêm altos valores relativos de densidade, de frequência e de dominância, enquanto muitas espécies apresentam poucos indivíduos e têm baixo IVI.

Tabela 2. Estimativa dos parâmetros fitossociológicos das espécies botânicas e do grupo de árvores mortas, na área de cerrado sensu stricto. Ao nível de amostragem a $0 \mathrm{~cm}$ do solo (CAS), ordenadas em ordem decrescente de valor de importância, em que $\mathrm{NI}=$ número de indivíduos; $\mathrm{DA}=$ densidade absoluta (número de indivíduos/ha); $\mathrm{DR}=$ densidade relativa $(\%)$; FrA = frequência absoluta (unidade de amostra/total de amostra); FrR = frequência relativa (\%); DoA = dominância absoluta $\left(\mathrm{m}^{2} / \mathrm{ha}\right)$; DoR = dominância relativa(\%) e IVI = índice de valor de importância (\%).

\begin{tabular}{|c|c|c|c|c|c|c|c|c|}
\hline Espécies & NI & DA & DR & FrA & FrR & DoA & DoR & IVI\% \\
\hline Myrcia fallax (Rich.) DC. & 137 & 274 & 15,12 & 100 & 2,2 & 2,11 & 5,69 & 7,67 \\
\hline $\begin{array}{l}\text { Protium heptaphyllum (Aubl.) } \\
\text { Marchand. }\end{array}$ & 63 & 126 & 6,95 & 100 & 2,2 & 4,72 & 12,71 & 7,29 \\
\hline Qualea multiflora Mart. & 68 & 136 & 7,51 & 100 & 2,2 & 1,45 & 3,9 & 4,54 \\
\hline Mortas & 38 & 76 & 4,19 & 100 & 2,2 & 2,65 & 7,15 & 4,52 \\
\hline Tapirira guianensis Aubl. & 28 & 56 & 3,09 & 100 & 2,2 & 2,94 & 7,93 & 4,41 \\
\hline Magonia pubescens A. St. -Hil. & 42 & 84 & 4,64 & 100 & 2,2 & 1,89 & 5,09 & 3,97 \\
\hline Qualea parviflora Mart. & 28 & 56 & 3,09 & 100 & 2,2 & 0,78 & 2,11 & 2,47 \\
\hline Luehea grandiflora Mart. & 18 & 36 & 1,99 & 100 & 2,2 & 1,18 & 3,19 & 2,46 \\
\hline Terminalia argentea Mart. & 15 & 30 & 1,66 & 80 & 1,76 & 1,46 & 3,94 & 2,45 \\
\hline Copaifera langsdorffii Desf. & 13 & 26 & 1,43 & 100 & 2,2 & 1,26 & 3,39 & 2,34 \\
\hline Coccoloba mollis Casar. & 25 & 50 & 2,76 & 100 & 2,2 & 0,69 & 1,86 & 2,27 \\
\hline Antonia ovata Pohl. & 19 & 38 & 2,1 & 80 & 1,76 & 1,05 & 2,83 & 2,23 \\
\hline Qualea grandiflora Mart. & 31 & 62 & 3,42 & 80 & 1,76 & 0,54 & 1,45 & 2,21 \\
\hline Byrsonima pachyphylla A. Juss. & 28 & 56 & 3,09 & 80 & 1,76 & 0,39 & 1,04 & 1,97 \\
\hline Vatairea macrocarpa (Benth.) Ducke & 17 & 34 & 1,88 & 100 & 2,2 & 0,6 & 1,61 & 1,90 \\
\hline Xylopia aromatica (Lam.) Mart. & 14 & 28 & 1,55 & 100 & 2,2 & 0,53 & 1,44 & 1,73 \\
\hline Emmotum nitens(Benth.) Miers. & 18 & 36 & 1,99 & 80 & 1,76 & 0,53 & 1,42 & 1,72 \\
\hline Astronium fraxinifolium Schott & 15 & 30 & 1,66 & 100 & 2,2 & 0,43 & 1,16 & 1,67 \\
\hline Roupala montana Aubl. & 14 & 28 & 1,55 & 100 & 2,2 & 0,42 & 1,14 & 1,63 \\
\hline Outras espécies & 275 & 550 & 30,33 & 2740 & 60,4 & 11,50 & 30,95 & 40,55 \\
\hline Total & 906 & 1812 & 100 & 4540 & 100 & 37,12 & 100 & 100 \\
\hline
\end{tabular}

Ao realizar a comparação dos dados deste trabalho com outros estudos (Tabela 3) pode-se perceber que o número de indivíduos por/ha variou de 165 a 1804 e área basal de $8,4 \mathrm{~m}^{2} /$ ha a $37,1 \mathrm{~m}^{2} /$ ha. Segundo Felfili et al. (1994), as amplitudes de densidade e área basal nos cerrados sensu stricto de Goiás e do Distrito Federal, variam entre 664 a

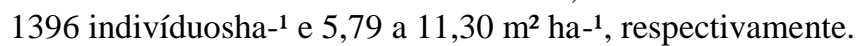

$\mathrm{O}$ valor de área basal encontrado nesse estudo foi elevado quando comparado a outros estudos que empregaram um critério de inclusão menos abrangente que o adotado nesse trabalho (CAS $\geq 10 \mathrm{~cm}$ ). É razoável, portanto, assumir que caso tivesse sido amostrado um critério de inclusão CAS $\geq 15,7 \mathrm{~cm}$ (geralmente empregado em cerrado sensu stricto), teríamos registrado uma área basal menor. A elevada área basal encontrada aproxima-se da fisionomia de cerrado denso, um subtipo de cerrado sensu stricto. Segundo Ribeiro e Walter (1998), o cerrado denso representa a forma mais densa e alta de cerrado sensu stricto, com vegetação predominantemente arbórea, de altura média entre cinco e oito metros, cuja cobertura varia entre $50 \%$ e $70 \%$. Dados estes que corroboram com a altura média registrada nesse estudo de 7,20m além de se aproximar com a altura encontrada no cerrado denso da RECOR - IBGE (ANDRADE et al., 2002), o que parece sustentar esta mesma classificação fisionômica para o fragmento estudado.

Ao se amostrar um hectare de cerrado sensu stricto espera-se encontrar um valor de riqueza no intervalo de 50 a 100 espécies (ASSUNÇÃO; FELFILI, 2004; BALDUINO et al., 2005; FELFILI; FAGG, 2007). Uma análise com 376 áreas de cerrado sensu lato ressaltou que é muito raro 
registrar mais do que 100 espécies/ha em um dado local (RATTER et al., 2003). Diante disso, embora o cerrado sensu stricto estudado possuir riqueza aparentemente elevada, principalmente se comparado a outras áreas de cerrado sensu stricto, dois fatores podem afirmar esta constatação: nosso estudo registrou 102 espécies ao abranger uma área menor do que muitos dos estudos com o qual foi comparado; além disso, as diferentes metodologias (formas de parcelas e critério de inclusão) empregadas nos estudos aqui comparados dificultam a interpretação de eventuais padrões que venham a ser detectados (BRIDGEWATER et al., 2004) (Tabela 3).

$\mathrm{O}$ índice diversidade de Shannon-Wienner (H') na área estudada foi de 3,70 , enquanto outros estudos no Tocantins,
Goiás e Maranhão (Tabela 3) possuem variação de H' entre 2,87 e 3,32. Embora o H' do cerrado sensu stricto da área estudada indique alta diversidade, o critério de inclusão adotado (mais inclusivo) certamente influenciou neste resultado, dificultando comparações com valores deste índice para outros locais (BRIDGEWATER et al.,2004; MAGURRAN, 2004).

O valor da equabilidade sugere que os indivíduos estiveram distribuídos de forma razoavelmente equânime entre as espécies, caracterizando baixa dominância ecológica. No entanto, elevada densidade de algumas espécies, como Myrcia fallax, Protium heptahyllum e Qualea multiflora.

Tabela 3. Comparação dos principais parâmetros fitossociológicos entre diversas áreas de cerrado. Fisio. = Fisionomia da área; NI = número de indivíduos; $\mathrm{AB}=$ área basal total por hectare; H' = índice de Shannon-Wiener; J' = equitabilidade de Pielou; Crit. = critério de inclusão (CAS-circunferência a altura do solo); Nsp = Número de espécies.

\begin{tabular}{|c|c|c|c|c|c|c|c|}
\hline Local de estudo & Fisio. & NI & $\mathrm{AB}$ & $\mathrm{H}^{\prime}$ & $\mathrm{J}$ ' & Crit. & Nsp \\
\hline Presente estudo & cerrado $s . s$. & 906 & 37,1 & 3,7 & 0,8 & $\mathrm{CAS} \geq 10$ & 102 \\
\hline Parque estadual do Lajeado, $\mathrm{TO}^{1}$ & cerrado s.s. & 1804 & 23,6 & 3,19 & 0,77 & $\mathrm{CAS} \geq 10$ & 60 \\
\hline Dueré - $\mathrm{TO}^{2}$ & cerrado s.s. & 249 & 9,24 & 3,31 & 0,86 & $\mathrm{CAS} \geq 15$ & 41 \\
\hline Rio Verde, $\mathrm{GO}^{3}$ & Cerrado s.s. & 165 & -- & 3,32 & 0,86 & $\mathrm{CAP} \geq 10$ & 29 \\
\hline Nordeste de GO e sudeste de $\mathrm{TO}^{4}$ & cerrado s.s. & 836 & 8,4 & 2,87 & 0,78 & $\mathrm{CAS} \geq 15,7$ & 87 \\
\hline Filadélfia, $\mathrm{TO}^{5}$ & cerrados.s & 789 & - & 3,32 & 0,83 & $\mathrm{CAS} \geq 0,5$ & 53 \\
\hline Carolina, $\mathrm{MA}^{5}$ & cerrado s.s. & 542 & -- & 3,04 & 0,77 & $\mathrm{CAS} \geq 0,5$ & 52 \\
\hline
\end{tabular}

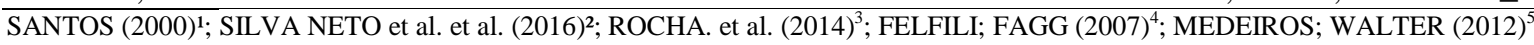

\section{CONCLUSÕES}

As famílias que apresentaram maiores valores de densidade e frequência e consequentemente Índice de valor de importância foram Myrtacea, Vochysiaceae, Burseraceae e Fabaceae, sendo as mais representativas floristicamente para o Brasil central.

Entre as espécies os maiores valores foram obtidas por Myrcia fallax (Rich.) DC, Protium heptaphyllum (Aubl) Marchand, Qualea multiflora Mart. e Tapirira guianensis Aubl.

Os valores obtidos para o índice de diversidade de Shannon-Wienner e equabilidade estão dentre os padrões encontrados no Cerrado.

\section{REFERÊNCIAS}

ANDRADE, L. A. Z.; FELFILI, J. M.; VIOLATTI, L. Fitossociologia de uma área de cerrado denso na RECORIBGE, Brasília-DF Fitossociologia de uma área de cerrado denso na RECOR-IBGE, Brasília-DF. Acta Bot. Bras. v.16, n.2, p.225-240, 2002.

ALMEIDA, S. P.; PROENÇA, C. E. B.; SANO, S.M.; RIBEIRO, J. F. Cerrado: espécies vegetais úteis. Planaltina: EMBRAPA-CPAC, 464p. 1998.

APG, ANGIOSPERM PHYLOGENY GROUP. An update of the Angiosperm Phylogeny Group classification for the orders and families of flowering plants: APG III. Bot. J. Linn. Soc., v.161, p.105-121. 2009.

ASSUNÇÃO, S. L.; FELIFILI, J. M. Fitossociologia de um fragmento de cerrado sensu stricto na APA do Paranoá, DF, Brasil. Acta Botanica Brasilica, v.18, p.903-909, 2004.
BALDUINO, A. P. D. C.; SOUZA, A. L. D.; MEIRA NETO, J. A. A.; SILVA, A. F. D.; SILVA JÚNIOR, M. C. D. Fitossociologia e análise comparativa da composição florística do cerrado da flora de Paraopeba-MG. Revista Árvore, v.29, n.1, p.25-34, 2005.

BRASIL. Ministério do Meio Ambiente. Mapeamento do Uso e Cobertura do Cerrado: Projeto Terra Class Cerrado 2013. Brasilia: MMA, v.1, p. 67, 2015.

BRASIL. Ministério do Meio Ambiente. O bioma Cerrado. Disponível em:<http://www.mma.gov.br/biomas /cerrado>Acesso em 10 jun. 2017.

BRIDGEWATER, S.; RATTER, J. A.; RIBEIRO, J. F. Biogeographic patterns, adiversity and dominance in the cerrado biome of Brazil. Biodiversity and Conservation, v.13, p.2295-2318, 2004.

CAVASSAN, O. Levantamento fitossociológico da vegetação arbórea da mata da Reserva Estadual de Bauru, utilizando o método dos quadrantes. Tese de Doutorado, Universidade Estadual de São Paulo, Rio Claro, 1982.

DURIGAN, G.; RATTER, J. A.; BRIDGEWATER, S.; SIQUEIRA, M. D.; FRANCO, G. A. D. C. Padrões fitogeográficos do cerrado paulista sob uma perspectiva regional. Hoehnea, v.30, p.39-51, 2003.

EMBRAPA, EMPRESA BRASILEIRA DE PESQUISA AGROPECUÁRIA. Sistema brasileiro de classificação de solos. Centro Nacional de Pesquisas de Solos. 3.ed. Rio de Janeiro, 2013. 353p.

FELFILI, J. M.; FILGUEIRAS, T. S.; HARIDASAN, M.; SILVA JÚNIOR, M. C.; MENDONÇA, R. C.; REZENDE, 
A. V. Projeto biogeografia do bioma Cerrado: vegetação e solos. Cadernos de Geociências, v.12, p.1-166, 1994.

FELFILI, J. M.; FAGG, C. W. Floristic composition, diversity and structure of the cerrado sensu stricto on rocky soils in northern Goiás and southern Tocantins, Brazil. Revista Brasileira de Botânica, v.30, p.375-385, 2007.

FELFILI, J. M.; da SILVA JÚNIOR, M. C.; SEVILHA, A. C.; FAGG, C. W.; WALTER, B. M. T.; NOGUEIRA, P. E.; REZENDE, A. V. Diversity, floristicandstructuralpatternsof cerrado vegetation in Central Brazil. PlantEcology, v.175, p.37-46, 2004.

FINA, G. B.; MONTEIRO, R. Análise da estrutura arbustivoarbórea de uma área de cerrado sensu stricto, município de Aquidauana - Mato Grosso do Sul. Revista Árvore. v.37, n.4, p.577-585, 2013.

FORZZA, R. C.; BAUMGRATZ, J. F. A.; BICUDO, C. D. M.; CARVALHO JÚNIOR, A. A.; COSTA, A.; COSTA, D. P.; MARTINELLI, G. Catálogo de plantas e fungos do Brasil. Rio de Janeiro: Instituto de Pesquisas Jardim Botânico do Rio de Janeiro, v.1 p.21-42, 2010.

GOODLAND, R. J. Análise ecológica da vegetação do cerrado. In: Ecologia do Cerrado. São Paulo: USP, p.61-171, 1979.

HARIDASAN, M.; ARAÚJO, G.M. Aluminium accumulating species in two forest communities in the cerrado region of the central Brazil. Manag: Forest Ecol. v.24, p.15-26, 1987.

HARIDASAN, M. Nutrição mineral de plantas nativas do cerrado. Revista Brasileira de Fisiologia Vegetal. v.12, p.5464, 2000.

LISTA DE ESPÉCIES DA FLORA DO. BRASIL. 2014 Jardim Botânico do Rio de Janeiro. Disponível em: $<\mathrm{h}$ ttp. floradobrasil. jbrj. gov. br/>. Acesso em 07 de jun de 2017, v. 31.

LOPES, W. P. Florística e fitossociologia de um trecho de vegetação arbórea no Parque Estadual do Rio Doce, Minas Gerais. Viçosa: UFV, v. 16, n.4, p. 443-456, 2002.

LORENZI, H. Árvores brasileiras: manual de identificação e cultivo de plantas arbóreas do Brasil, vol. 1. 4 ed. Nova Odessa: Instituto Plantarum, 2002.

MACHADO, R. B.; RAMOS NETO, M. B.; PEREIRA, P. G. P.; CALDAS, E. F.; GONÇALVES, D. A.; SANTOS, N. S.; STEININGER, M. Estimativas de perda de área do cerrado brasileiro. Conservação Internacional, Brasília, 2004.

MAGURRAN, A. E. Measuring biological diversity. Blackwell Science Ltd., Oxford, 2004. 256p.

MARTINS, F. R. Estrutura de uma floresta mesófila. Campinas: Editora da UNICAMP, 1991.
MUELLER-DOMBOIS, D. Y.; ELLENBERG, M. Aims and methods in vegetation ecology. New York: John Wiley \& Sons, 1974. 547p.

NERI, A. V.; MEIRA NETO, J. A. A.; SILVA, A. F. D.; MARTINS, S. V.; BATISTA, M. L. Análise da estrutura de uma comunidade lenhosa em área de cerrado sensu stricto no município de Senador Modestino Gonçalves, norte de Minas Gerais, Brasil. Revista Árvore, v.31, n.1, p.123-134, 2007.

NETTESHEIM, F. C. Estrutura e florística do estrato arbóreo no cerrado sensu stricto de Buritis, Minas Gerais, Brasil. Rodriguésia-Instituto de Pesquisas Jardim Botânico do Rio de Janeiro, v. 61, n. 4, 2010.

OLIVEIRA, P. E. A. M. Fenologia e Biologia Reprodutiva das Espécies de Cerrado. In: SANO, S.M.; ALMEIDA S.P.; RIBEIRO J.F. Cerrado: ecologia e flora. Planaltina: EMBRAPA-CPAC, p.273-290, 2008.

POLHILL, R. M.; RAVEN, P. H.; STIRTON, C. H. Evolution and systematics of the Leguminosae. In: POLHILL R.M.; RAVEN P.H. Advances in legume systematics. Kew Garden, 1981.

RATTER, J. A.; BRIDGWATER, S.; RIBEIRO, J. F. Analysis of the floristic composition of the braziliancerrado vegetation III: Comparison of the woody vegetation of 376 areas. Edinburgh Journal of Botany, v.60, n.1, p.57-109, 2003.

RIBEIRO, J. F.; WALTER, B. M. T., SANO, S. M.; ALMEIDA, S. D. Fitofisionomias do Bioma Cerrado. Cerrado, ambiente e flora. Planaltina: Embrapa - CPAC, p.89-166. 1998.

ROCHA, E.; REYS, P.; SILVA, P. O.; SOARES, M. P. Florística e fitossociologia de um fragmento de cerrado no sudoeste de Goiás Revista Global Science Technology. v. 7, n. 3, p.110-118, 2014.

SANO, S. P.; ALMEIDA, J. F. R. Cerrado: Ecologia e Flora. Embrapa cerrados/Embrapa. Brasília, DF, 2008. p.151- 212.

SANTOS, L. D.; MARMONTEL, C. V. F., MARTINS, T.; MELO, A. G. C.. Fitossociologia de cerrado sensu stricto localizado no Município de Carbonita-MG. Revista Científica Eletrônica de Engenharia Florestal, v. 15, n. 1, p. 77-90, 2010.

SEPLAN. Atlas do Tocantins: subsídios ao planejamento da gestão territorial, Palmas. Atual, v.6, 2012.

SHEPHERD, G. J. FITOPAC 2: manual do usuário. Campinas: UNICAMP, 2010.91p.

SILVA JÚNIOR, M. C. + 100 árvores do cerrado -Matas de Galeria: guia de campo. Brasília. Ed. Rede de sementes do cerrado, 288 p. 2009.

SILVA JÚNIOR, M. C. 100 árvores do cerrado- sentido restrito: guia de campo. Brasília. Ed. Rede de sementes do cerrado, 304 p. 2012. 
SILVA, L. A.; SOARES, J. J. Levantamento fitossociológico em um fragmento de floresta estacional semidecídua, no município de São Carlos, SP. Acta bot. bras., v.16, n.2, p.205216, 2002.

SILVA NETO, V. L.; de OLIVEIRA, A. L.; de SOUZA FERREIRA, R. Q.; de SOUZA, P. B.; VIOLA, M. R. Fitossociologia e distribuição diamétrica de uma área de cerrado sensu stricto, Dueré-TO. Revista de Ciências Ambientais, v. 10, n. 1, p. 91-106, 2016.

SILVA, R. B. M.; FRANCELINO, M. R.; MOURA, P.A.; MOURA, T. A.; PEREIRA, M. G.; OLIVEIRA, C. P. Relação solo/vegetação em ambiente de cerrado sobre influência do grupo Urucuia. Ciênc. Florest., v.25, n.2, pp.363-373, 2015.
SOUZA FERREIRA, R. Q.; CAMARGO, M. O.; SOUZA, P. B.; ANDRADE, V. C. L. Fitossociologia e estrutura diamétrica de um cerrado sensu stricto, Gurupi-TO. Revista Verde de Agroecologia e Desenvolvimento Sustentável, v. 10, n. 1, p. 229-235, 2015.

STRUFFALDI-DE-VUONO, Y. Fitossociologia do estrato arbóreo da floresta da Reserva do Instituto de Botânica (São Paulo, SP). Tese de Doutorado, Universidade de São Paulo, São Paulo, 1985.

TABANEZ, A. J.; VIANA, V. M.; DIAS, A. S. Consequências da fragmentação e do efeito de borda sobre a estrutura, diversidade e sustentabilidade de um fragmento de floresta de planalto de Piracicaba, SP. Rev. Bras. Biol. v.57, n.1, p.47-60, 1997. 